\title{
Modeling of Longitudinal Factors Under-Age Five Children Body Mass Index at Bahir Dar Districts: First Order Transition Model
}

\author{
Alebachew Abebe Alemu ${ }^{1}$
}

Received: 28 February 2018 / Revised: 25 April 2019 / Accepted: 8 May 2019 / Published online: 23 May 2019 (c) The Author(s) 2019

\begin{abstract}
The body mass index (BMI) is calculated as weight in kilograms divided by square height in meters $\left(\frac{\mathrm{kg}}{\mathrm{m}^{2}}\right)$. Its formula was developed by Belgium Statistician Adolphe Quetelet, and was known as the Quetelet Index (Adolphe Quetelet in BMI formula was developed. Belgium Statistician, 1796-1874. http://www.cdc.gov/healthyweight/ assessing/bmi/childrens_bmi/about_childrens_bmi.htm). It provides a reliable indicator of body fatness for most people and is used to screen weight categories that may lead to health problems. BMI is an internationally used measure of health status of an individual. This study was modeling of longitudinal factors under-age five children BMI at Bahir Dar Districts using First Order Transition Model. This study was based on data from 1900 pre four visits (475 per individual) children enrolled in the first 4 visits of the 4-year Longitudinal data of children in Bahir Dar Districts. First order transition model was used to describe the relationships between children BMI and some covariates accounting for the correlation among the repeated observations for a given children. There were statistically significant $(P$ value $<0.05)$ difference among children BMI variation with respect to time, Sachet (plump nut), age, residence, Antiretro-Viral Therapy, diarrhea and pervious BMI. But, fever, cough, Mid-Upper Arm Circumference and sex were statistically insignificant ( $p$ value $>0.05)$ effect on children BMI. According to the findings of this study about $29.28 \%$ were normal weight, $67 \%$ were under weight, $2.52 \%$ were overweight and only $1.21 \%$ were obesity. Consequently, the study suggests that concerned bodies should focus on awareness creation to bring enough food to under-age five children in Bahir Dar Districts especially in rural areas.
\end{abstract}

Keywords BMI · Intra-class correlation coefficient · Transition model · Trivial solution · Working covariance matrix

Alebachew Abebe Alemu

aleb.abebe@yahoo.com; aleb.abebe19@gmail.com

1 Department of Statistics, College of Computing and Informatics, Haramaya University, P.O. Box 138, Dire Dawa, Ethiopia 


\section{Introduction}

The body mass index (BMI) was developed by Belgium Statistician Adolphe Quetelet [1]. BMI is calculated as weight in kilograms divided by square height in meters. Centers of Disease Control and Prevention (CDC) and the American Academy of Pediatrics (AAP) recommend the use of BMI to screen for overweight and obesity in children beginning at the age of 2 years. BMI is used to screen for obesity, overweight, healthy weight or underweight. However, BMI is not a diagnostic tool. According to WHO technical report series 854:9, the BMI range less than 18.5 indicates underweight, 18.5-25 indicates normal weight, 25-30 indicates over weight and over 40 indicates obesity.

In recent years, overweight and/or obesity among children have emerged as a global epidemic [18]. The fundamental cause of obesity and overweight is an energy imbalance between calories consumed and calories expended. Globally there has been an increased intake of energy-dense foods that are high in fat, salt and sugars but low in vitamins, minerals and other micronutrients and decrease in physical activity due to the increasingly sedentary nature of many forms of work, changing modes of transportation and increasing urbanization [17]. In 2005, the WHO reported that at least 400 million adults were obese and at least 20 million children under the age of 5 years are overweight globally. WHO further projects that by 2015 approximately 2.3 billion adults were overweight and more than 700 million were obese. There is still much uncertainty related to the causes and underlying physiological mechanisms of obesity. Recent scientific findings are able to show that long-term risk of obesity and related disorders begin very early in life [4].

In Africa, despite a high prevalence of under nutrition, the prevalence of overweight is increasing at an alarming rate. It is estimated that $25-60 \%$ of urban women are overweight [5]. According to Tanzania Health Research (2006) conducted in Simanjiro District, $82 \%$ of the adolescents had normal health status while $0.8 \%$ were overweight for their ages, $14.0 \%$ were moderately wasted and $3.2 \%$ were severely wasted. This could be explained by the change in the life style factors of the society. Moreover, the study was conducted on the prevalence of overweight and/or obesity in Sudan which was $14 \%$ [14].

In Ethiopia, study was conducted in Addis Ababa in 2007 reported that the prevalence of overweight and obesity on elementary school students were $7.6 \%$ and $0.9 \%$ respectively [2]. Similarly, 2014 in Addis Ababa conducted that prevalence of overweight, obesity and associated factors among high school adolescents in Arada sub-city were $72.1 \%$ normal-weight, $18.5 \%$ underweight, $8.6 \%$ overweight and $0.8 \%$ obesity [3]. This might be due to the food eaten in Sudan were highly energy dense foods and there is also frequent eating habits while in Ethiopia mostly eaten foods are fibers and cereals, three times a day. Overweight and obesity during childhood increases the risk for the development of non-communicable diseases and predisposes the individual to the development of overweight, obesity, cardiovascular disease and metabolic and other disorders in childhood [3].

Children BMI are a serious threat to the governments' effort to meet the growth and transformation plan (GTP) two. Further, there are also few local researchers who have done on the issue, however, their method of analysis has descriptive in nature 
and limited to examine underline factors association between children BMI status with certain BMI-related covariate. In addition, most of those studies are based on small-scale survey data that obtained from certain district. There had been no detailed scientific study on this thematic area especially in Bahir Dar Districts, Ethiopia. The study will help to fill the gap of knowledge on BMI of children and the output will help to recommend bodies for primary prevention. Now, this study was planned to identify the basic risk factors under-age five children BMI were compressed the secondary data of those children BMI by using first order transition model (FOTM).

The main objective of this study was to model longitudinal factors under-age five children BMI by using the best robust first order transition model. The motivation of this paper was applied as useful a statistical approach like transition models to a topic of significant public health importance. The research gaps for this paper is children BMI depend on previous BMI so closely linked to determine the present outcomes that examining the factors influencing children BMI was crucial to minimizing potential negative impact. In this study, model development procedures were AIC and BIC and also forward selection was used for model selection.

\section{Materials and Methods}

This study was based on data from 475 children enrolled in the first four visits of the four year longitudinal study of children BMI using the data of Bahir Dar Districts working collaboration with Save the Children. The study area was located in Bahir Dar, Ethiopia and serve as a capital city of Amhara regional state, $563 \mathrm{~km}$ far away from Addis Ababa in North West direction. This study was retrospective study on longitudinal data setting design that go back in time to assess exposure to known the trend and modeling of longitudinal factors on children BMI. The different socioeconomic status, demographic, disease types and biological/clinical characteristics were collected repeatedly in four waves between the years on January 2012 to 2016. Each repeated measures was conducted within one month interval in the study period. In this study both time invariant and variant covariates were employed. The first wave was conducted on January 2012 to 2013 within one month periods in 4 repetitions. Similarly, the second, third and fourth waves were conducted on January 2013 to 2014, 2014 to 2015 and 2015 to 2016 were presented respectively.

\subsection{Variables Considered in the Study}

Children BMI health status was considered as the response variable. BMI (in a standardized form) was used as a continuous variable to maximize the amount of information available in the data set. And also, Explanatory variables (Covariates) are time of child treatment, age of child, child's sex (female, male), amount of sachet, place of residence (rural, urban), cough status (yes, no), diarrhea status (yes, no), fever status (yes, no), amount of Mid-Upper Arm Circumference (MUAC) and Antiretro-Viral Therapy (ART) treatment (on ART, on pre-ART and no ART). 


\subsection{Inferential Statistics}

\subsubsection{Methods of Statistical Analysis}

Longitudinal data is a special case of repeatedly measured data, the observations are not independent and are characterized as having both between-subject and within-subject variation, time dependent covariates and missing data [6]. The variance covariance structure does not have to be independent. Data can be balanced or each subject does need to have the same number of observations per subject and repeated measurements have equal time intervals. Furthermore, mixed-effects modeling have become increasingly popular, more accessible and good in missing data handling through statistical software such as SAS Version-9.2 [15].

\subsubsection{Exploring Data Analysis}

Before model fit, it's illustrating relevant raw data as much as possible, identifying longitudinal patterns that may be of interest and identifying outliers or unusual observations [8]. Data exploring was extremely helpful as additional tool in the selection of appropriate models. The aspects of the data that shall be included are Individual Profiles, Exploring Mean Structure, Exploring Variance Structure, Exploring the Random Effects and Exploring the Correlation Structure were used for this study. There are four main types covariance models were used for used for this study.

\subsubsection{Compound Symmetry (CS) Covariance Models}

$$
\mathrm{CS}=\sum=\sigma^{2}\left[\begin{array}{cccc}
1 & \rho^{2} & \cdots & \rho^{2} \\
\vdots & \vdots & \ddots & \vdots \\
\rho^{2} & \rho^{2} & \cdots & 1
\end{array}\right]
$$

\subsubsection{Autoregressive Structure of order one [AR(1)] Covariance Models}

$$
\operatorname{AR}(1)=\sum=\sigma^{2}\left[\begin{array}{cccc}
1 & \rho & \cdots & \rho^{\mathrm{m}-1} \\
\vdots & \vdots & \ddots & \vdots \\
\rho^{\mathrm{m}-1} & \rho^{\mathrm{m}-2} & \cdots & 1
\end{array}\right]
$$

\subsubsection{Toeplitz (TOEP) Covariance Models}

$$
\operatorname{TOEP}=\sum=\sigma^{2}\left[\begin{array}{cccc}
1 & \rho_{1}^{2} & \cdots & \rho_{\mathrm{m}-1}^{2} \\
\vdots & \vdots & \ddots & \vdots \\
\rho_{\mathrm{m}-1}^{2} & \rho_{\mathrm{m}-2}^{2} & \cdots & 1
\end{array}\right]
$$




\subsubsection{Unstructured (UN) Covariance Models}

$$
\mathrm{UN}=\sum=\left[\begin{array}{cccc}
\sigma_{11} & \sigma_{12} & \cdots & \sigma_{1 \mathrm{~m}} \\
\vdots & \vdots & \ddots & \vdots \\
\sigma_{\mathrm{m} 1} & \sigma_{\mathrm{m} 2} & \cdots & \sigma_{\mathrm{mm}}
\end{array}\right]
$$

\subsubsection{Transition Model}

A transition model has also been used and is a function of covariates and of past responses [8]. Conditional distribution of each $Y_{i j}$ are modeled directly in terms of preceding $Y_{i 1},---, Y_{i j-1}$, those previous children BMI considered as covariates (explanatory variables). Good for prediction of present children BMI based on the previous children BMI. In this study $Y_{(i j)}$ is present children BMI and $X_{i j}$ are covariates of child $i$ at time $t_{i j}$, and also affected by previous children BMI.

$$
E\left(\frac{Y_{i j}}{\text { previous } B M I}\right)=\mu_{i j}=X_{i j}^{\prime} \beta+Y_{(i j)}^{\prime} \alpha \text {; where } Y_{(i j)}=\left(Y_{i 1},---, Y_{i j-1}\right) \text {, }
$$
Because, the data nature is continuous and it follows a normal distribution. Typically, $\frac{Y_{i j}}{\text { pervious } B M I}$ is assumed to have an exponential family distribution with variances. $\operatorname{Var}\left(\frac{Y_{i j}}{\text { previous BMI }}\right)=\phi V\left(\mu_{i j}\right)$; Where $\mathrm{V}$ a known function and the scale parameter are $\phi$ may also depend on some covariates. Construct likelihood as product of conditional distributions, usually assuming restricted form of dependence, for example: $f_{k}\left(\frac{y_{i j}}{y_{i 1}},---, y_{i j-1}\right)=f_{k}\left(\frac{y_{i j}}{y_{i j-1}}\right)$ and condition on $y_{i 1}$ as model does not directly specify $f_{i 1}\left(y_{i 1}\right)$. Correlation among $Y_{i 1},---, Y_{i n_{i}}$ exists because the past children BMI values explicitly influence the present children BMI. i.e. $\operatorname{cor}\left(\frac{Y_{i j}}{\text { previous } B M I}\right)=\rho\left(\mu_{i j}\right.$, previous $\left.B M I, \alpha\right)$; where $\rho$ is a known function and the correlation parameters $\alpha$ may depend on covariates.

\subsubsection{Methods of Parameter Estimation}

Estimating equations for $\beta$ in a longitudinal data for GLM:

$$
\begin{aligned}
& S\left(\beta_{j}\right)=\sum \frac{\partial \mu_{i}}{\partial \beta_{j}} V_{i}^{-1}\left(Y_{i}-\mu_{i}\right)=0 \\
& S\left(\beta_{j}\right)=\sum \frac{\partial \mu_{i}}{\partial \beta_{j}} V_{i}^{-1}\left(Y_{i}-\mu_{i}\right)=0
\end{aligned}
$$

where $j=1,---, p$ and $V_{i}=\operatorname{Var}\left(Y_{i}\right)$.

In vector-matrix notation:

$$
S(\beta)=D_{\mu i}^{\prime} V^{-1}(Y-\mu)=0
$$

$D_{\mu \beta}$ is an $n \times p$ matrix with $i j$ th element $\frac{\partial \mu_{i}}{\partial \beta_{j}} ; \mathrm{V}$ is an $n \times n$ diagonal matrix with non-zero elements proportional to $\operatorname{Var}\left(Y_{i}\right) ; Y$ and $\mu$ are n-element vectors with elements $Y_{i}$ and $\mu_{i}$. 
In the longitudinal setting they are replaced by $n_{i}$ element vectors $Y_{i}$ and $\mu_{i}$, associated with $i$ th subject. Corresponding matrices $V_{i}(\alpha)=\operatorname{Var}\left(Y_{i}\right)$ are no longer diagonal.

Estimating equations for complete set of data, $Y\left(y_{1},---, y_{m}\right)$ :

$$
S(\beta)=\sum_{i=1}^{m}\left\{D_{\mu \beta}\right\}^{\prime}\left\{V_{i}(\alpha)\right\}^{-1}\left(Y_{i}-\mu_{i}\right)=0
$$

Large-sample properties of resulting estimates $\hat{\beta}$ :

$$
\sqrt{(m)}(\hat{\beta}-\beta) \sim \operatorname{MVN}\left(0, I_{0}^{-1}\right)
$$

where $I_{0}=\sum_{i=1}^{m}\left\{D_{\mu 1 \beta}\right\}^{\prime}\left\{V_{i}(\alpha)\right\}^{-1} D_{\mu 1 \beta}$, what we have to do when variance matrices $V_{i}(\alpha)$ are unknown? The working covariance matrix:

$$
S(\beta)=\sum_{i=1}^{m}\left\{D_{\mu 1 \beta}\right\}^{\prime}\left\{V_{i}^{*}(\alpha)\right\}^{-1}\left(Y_{i}-\mu_{i}\right)=0
$$

$V_{i}^{*}($.$) is a guess at the covariance matrix of Y_{i}$ called the working covariance matrix. Result (6) on distribution of $\hat{\beta}$ now modified to:

$$
\sqrt{(m)}(\hat{\beta}-\beta) \sim \operatorname{MVN}\left(0, I_{0}^{-1} I_{1} I_{0}^{-1}\right)
$$

where $I_{0}=\sum_{i=1}^{m}\left\{D_{\mu i \beta}\right\}^{\prime}\left\{V_{i}(\alpha)\right\}^{-1} D_{\mu i \beta}$ and $I_{1}=\sum_{i=1}^{m}\left\{D_{\mu i \beta}\right\}^{\prime}\left\{V_{i}^{*}(\alpha)\right\}^{-1}$ $\operatorname{Var}\left(Y_{i}\right)\left\{V_{i}^{*}(\alpha)\right\}^{-1} D_{\mu i \beta}$.

The relationship between variance matrix $\left[V_{i}(\alpha)\right]$ and working covariance matrix $\left[V_{i}^{*}().\right]$ were used to calculate the working covariance matrix in the model as follow:

(i) Result (7) reduces to if $V_{i}^{*}()=.V_{i}($.

(ii) Estimator $\hat{\beta}$ is consistent event if $V_{i}^{*}()=.V_{i}$ (.) and

(iii) To calculate an approximation to $I_{1}$, replace

$$
\operatorname{Var}\left(Y_{i}\right) \text { by }\left(Y_{i}-\hat{\mu}_{i}\right)\left(Y_{i}-\hat{\mu}_{i}\right)^{\prime} \text { where } \hat{\mu}_{i}=\mu_{i}(\hat{\beta})
$$

The procedures were applied to make trivial estimator of working covariance matrix to calculate coefficient parameters are as follow:

(i) Numbers of subjects (n) are smaller than number of repetition times (m)

(ii) The same model for $\mu_{i}$ fitted to groups of subjects and

(iii) Observation times common to all subjects.

Otherwise, a bad choice of $V_{i}^{*}($.) does affect efficiency of $\hat{\beta}$. In transition model, explanatory variables and previous children BMI are treated symmetrically as predictors of the present children BMI. Hence, as the time dependence model changes, so might inferences about sensitivity of inferences with respect to time dependence assumptions. 


\subsubsection{Assumptions of Transition Model}

Most commonly using transition model is FOTM could have the form:

$$
\begin{gathered}
Y_{i j}=X_{i j}^{\prime} \beta+\epsilon_{i j} \\
\epsilon_{i j}=\alpha \epsilon_{i j-1}+Z_{i j}
\end{gathered}
$$

where $Z_{i j}$ are independent mean-zero with variance $\delta^{2}$, and the process is initiated by:

$$
\begin{aligned}
& \in_{i 0} \sim N\left\{0, \frac{\delta^{2}}{\left(1-\alpha^{2}\right)}\right\} \text {. This model can be re-expressed as: } \\
& \qquad Y_{i j}=X_{i j}^{\prime} \beta+\alpha\left(Y_{i j-1}-X_{i j-1}^{\prime} \beta\right)+Z_{i j}
\end{aligned}
$$

Equations (10) and (11) imply that $E\left(Y_{i j}\right)=X_{i j}^{\prime} \beta$, so this form of linear transition model has coefficients which also have a marginal interpretation. This model is equivalent to a marginal model with an exponential autocorrelation function.

Econometricians use a different formulation of transition model:

$$
Y_{i j}=X_{i j}^{\prime} \beta+\alpha Y_{i j-1}+Z_{i j}
$$

The response is regressed on the previous outcome itself without adjusting for its expectation, like Eq. (11) does. Equation implies that $E\left(Y_{i j}\right)=\sum_{r=0} \alpha^{r} X_{i j-r}^{\prime} \beta$, so that $\beta$ does not have a marginal interpretation. The interpretation of $\beta$ depends on the assumed form of the autocorrelation model. Finally, the conditional distribution of first order transition model is:

$$
E\left(\frac{Y_{i j}}{Y_{i j-1}}\right)=X_{i j}^{\prime} \beta+\alpha Y_{i j-1}
$$

\section{Results and Discussion}

\subsection{Results}

\subsubsection{Descriptive Statistics Analysis}

From the total of 475 children BMI under age five the minimum age were 0.17 month, maximum age were 5.00 years, and the average age of these children was 2.01 and the standard deviation of 1.17. MUAC status had the mean of 12.20 and the standard deviation of 0.90 . In similar fashion, the amount of pump net (sachet) consumed by children was the mean of 36.78 and its standard deviation was 13.74 (Table 1). 
Table 1 Descriptive statistics for continuous covariates

\begin{tabular}{llllc}
\hline Variables & Minimum & Maximum & Mean & \multicolumn{1}{l}{ SD } \\
\hline Age & .17 & 5.00 & 2.0096 & 1.16726 \\
MUAC status & 4.2 & 14.6 & 12.197 & .9015 \\
Plump net & 15 & 90 & 36.78 & 13.740 \\
\hline
\end{tabular}

Of the total 475 number of children, at the first time of measurement cough status indicates that $41.46 \%$ of children were coughed and the remaining $58.95 \%$ were noncoughed. $34.81 \%$ of children were have diarrhea and $65.19 \%$ of children were haven't diarrhea. Moreover, $51.11 \%$ of children have fever and $48.89 \%$ haven't fever in the first four visits. The remaining covariates interpretations and discussions will be made in the same fashion (Table 2).

Assessing health status of children BMI measured by Sex had $26.49 \%$ of males and $31.19 \%$ females were normal weight status; $69.93 \%$ of males and $65 \%$ of females were underweight status; where by $1.98 \%$ males and $2.88 \%$ females were overweight and $1.61 \%$ of males and $0.93 \%$ of females were obesity. Moreover, children based on place of residence, $65.37 \%$ rural children and $68.35 \%$ left behind urban children were underweight; while $2.66 \%$ rural children and $2.39 \%$ urban children were with overweight and $0.89 \%$ of rural children and $1.47 \%$ of urban children were with obesity. The remaining covariates interpretations and discussions will be made in the same fashion (Table 3).

Based on WHO criteria from 475 number of children examined around $29.28 \%$ were normal weight status, $67 \%$ were under weight, $2.52 \%$ were overweight and only $1.21 \%$ were obesity. The time of treatment for children increased from time 1 to time 4 , then the number of children who had normal weight status were increased over time and the number of children who had underweight status were decreased over time. Mean of children BMI tends to increase over time and similarly variance of children BMI tends to increase over time. Generally, this study seems to have a problem of underweight than overweight and obesity in percentage (Table 4).

\subsubsection{Explanatory Data Analysis}

3.1.2.1 Individual Profile Plots It provides some information between children BMI variability and illustrate that there is a change among children BMI over time. Likewise, it appears that most of the children are gaining BMI over time and the variability of the children BMI seems smaller at the beginning compared to the end (Fig. 1).

3.1.2.2 Exploring Mean Structure The mean profile plot gives clue to determine the type of time effect on BMI and the time seems to have almost linear effect on BMI (Fig. 2).

3.1.2.3 Exploring Variance Structure Plot of variance appears that the observed variance was not constant through time evolution and seems variability of children BMI tends to increase over time (Fig. 3a). The variability of BMI for males looked higher than that of females through time. The slope of female children BMI had higher rate than that of male children through time evolution (Fig. 3b). 


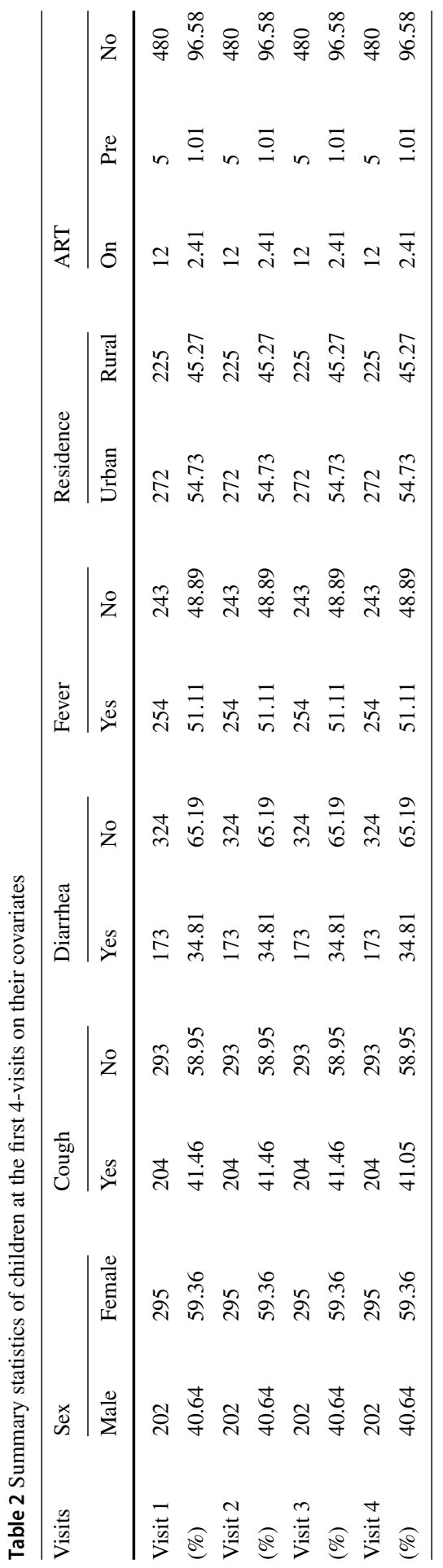




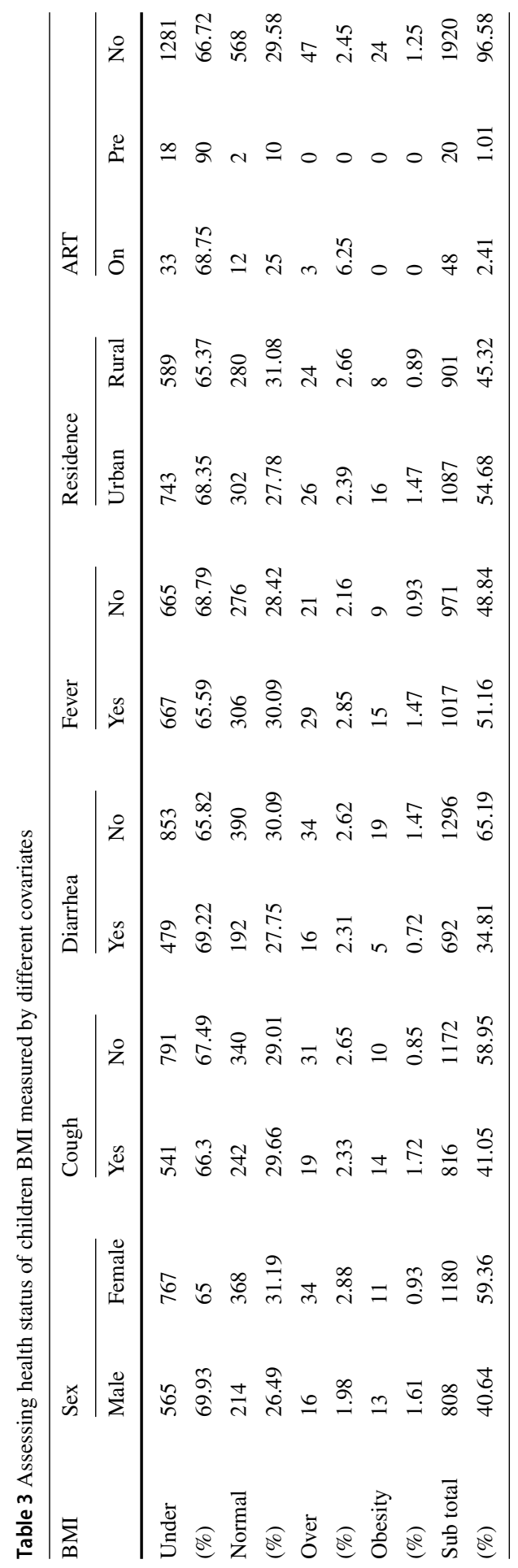


Table 4 Assessing health status of children BMI measured over time

\begin{tabular}{|c|c|c|c|c|c|c|}
\hline \multirow[t]{2}{*}{ Time } & \multicolumn{4}{|c|}{ Health Status } & \multicolumn{2}{|l|}{ BMI } \\
\hline & Normal W. & Obesity & Over W. & Under W. & Mean & Variance \\
\hline Time1 & 24 & 0 & 2 & 471 & 14 & 6.94 \\
\hline$(\%)$ & 4.12 & 0 & 4 & 35.36 & & \\
\hline Time2 & 179 & 1 & 6 & 311 & 17.19 & 11.53 \\
\hline$(\%)$ & 30.76 & 4.17 & 12 & 23.35 & & \\
\hline Time3 & 186 & 5 & 18 & 288 & 17.82 & 14.88 \\
\hline$(\%)$ & 31.96 & 20.83 & 36 & 21.22 & & \\
\hline Time4 & 193 & 18 & 24 & 262 & 18.81 & 24.69 \\
\hline$(\%)$ & 33.16 & 75 & 48 & 19.67 & & \\
\hline Sub total & 582 & 24 & 50 & 1332 & & \\
\hline$(\%)$ & 29.28 & 1.21 & 2.52 & 67 & & \\
\hline
\end{tabular}

Fig. 1 Individual profile plot

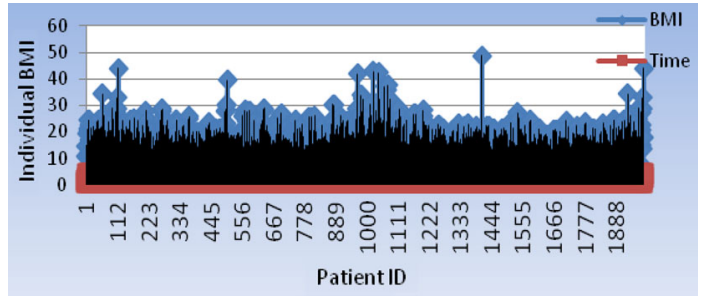

Fig. 2 Mean profile plots

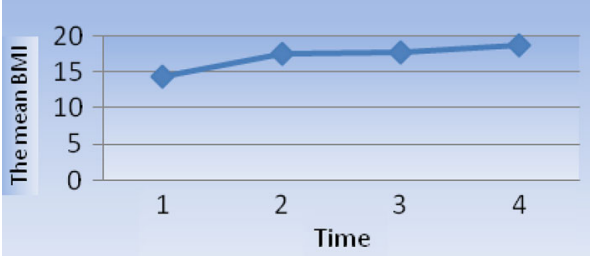

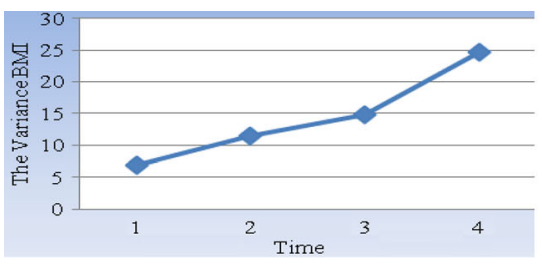

(a)

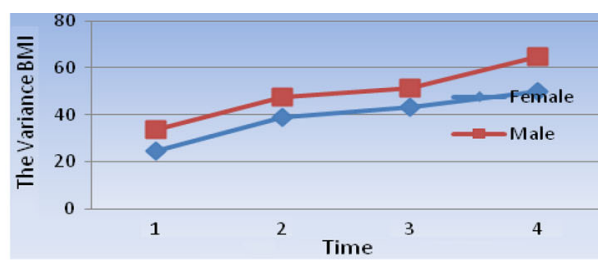

(b)

Fig. 3 Observed variance and variability of children BMI by sex 


\subsubsection{Checking Assumption for the Final Model}

Individual specific residual plots for fitted model designates that the residuals are centered at zero. That is, $\mathrm{E}\left(\varepsilon_{i j}\right)=0$, but the variability changes with group (Fig. $4 \mathrm{a}$ in Appendix). Since there were only four observations per individual, we couldn't rely too much on the individual box plots for inference about the within-group variances. Examining the plot of the standardized residuals versus fitted values by gender appears to show that the variability in BMI measurements were slightly greater among males than among females (Fig. 4b in Appendix). However, with in each gender the variability was somewhat constant which might imply heteroscesdasticity. The adequacy of the heteroscedastic fit has been assessed by examining plots of the standardized residuals versus the fitted values by sex. The standardized residuals in each sex now have about the same variability, but not exactly the same and the qq normal plot had shown that random errors were approximately normally distributed and symmetric with respect to zero. Therefore, the normality assumption was shown (Fig. 4c in Appendix).

\subsubsection{Assessing the Assumption on the Random Effects}

Basically, qq-norm normal plot of estimated random effects were used for checking marginal normality and identifying outliers whereas pairs scatter plot matrix of the estimated random effects were used for identifying outliers and checking the assumption of homogeneity of the random effects covariance matrix (Fig. 4d, $\mathrm{f}$ in Appendix). The heteroscedastic model accommodates the impact of the outlying observations in the within-group variances estimation and this accommodation reduces the estimated between group variability, thus increasing the degree of shrinkage in the random effects estimates. Box plots of Sex for heterosedastic model did not suggest any departures from the assumption of homogeneity of the random effects distribution (Fig. 4e in).

\subsubsection{First Order Transition Model}

Mean profile plots have suggested that time has nearly linear effect on BMI progression over time (Fig. 2). Thus, first order transition model with linear time effect was fitted as:

$$
\begin{aligned}
B M I_{i j}= & \beta_{0}+\beta_{1} T_{i j}+\beta_{2} A_{i}+\beta_{3} P_{i}+\beta_{4} M_{i}+\beta_{5} S_{i}+\beta_{6} C_{i}+\beta_{7} D_{i}+\beta_{8} F_{i} \\
& +\beta_{9} A R_{i}+\beta_{10} R_{i}+\beta_{11} P_{i} * T_{i j}+\beta_{12} S_{i} * T_{i j}+\beta_{13} P_{i} * T_{i j} * S_{i} \\
& +\beta_{14} M_{i} * T_{i j}+\beta_{15} A_{i} * T_{i j}+\beta_{16} D_{i} * R_{i}+\alpha B M I_{-1}+\varepsilon_{i j}
\end{aligned}
$$

where $B M I_{i j}=$ Body Mass Index on $i$ th children on $j$ th measurement; $T_{i j}=$ Time at which $i$ th children on $j$ th measurement, $i=1,---, 1900$ and $j=1,2$, 3, 4.; $A_{i}=$ Age at $i$ th children; $P_{i}=$ Amount of plump nut given for $i$ th children; $M_{i}=$ MUAC status for $i$ th children; $S_{i}=$ Sex of $i$ th children (Male, Female); $C_{i}=$ Cough status $i$ th children (Yes, No); $D_{i}=$ Diarrhea status $i$ th children (Yes, No); $F_{i}$ $=$ Fever status $i$ th children (Yes, No); $A R_{i}=$ ART treatment $i$ th children (On ART, on pre-ART, No ART); $R_{i}=$ Residence of $i$ th children (Urban, Rural); $P_{i} * T_{i j}, S_{i} * T_{i j}$, $M_{i} * T_{i j}, \quad A_{i} * T_{i j}=$ Interaction terms with time; $P_{i} * T_{i j} * S_{i}=$ Interaction terms 
Table 5 Selection of best random effects based on intercept, slope and both cases

\begin{tabular}{llllllll}
\hline & Model & df & AIC & BIC & loglik test & L.ratio & $p$ value \\
\hline Both & 1 & 19 & 9504.7 & 9505.1 & 9464.7 & 847.76 & $<0.0001$ \\
Slope & 2 & 19 & 9683 & 9685.3 & 9607.7 & & \\
Intercept & 3 & 19 & 9640.8 & 9685.4 & 9640.8 & & \\
\hline
\end{tabular}

between plump nut and sex with time; $D_{i} * R_{i} * T_{i j}=$ Interaction between diarrhea and residence over time; $B M I_{-1}=$ the previous children body mass index; $\beta_{0}=$ Over all intercepts; $\beta_{1},---, \beta_{16}=$ Coefficients of fixed effect; $\alpha=$ Coefficient of previous BMI; $\varepsilon_{i j}=$ Random error term

3.1.5.1 Random Effect Term Selection To select random effect to the model with only intercept, with only slope and with intercept and slope have been fitted and compared. An appropriate random effect to the model was selected by using likelihood ratio test. The small p-value indicates that, we can reject model 2 in favor of model 1; we prefer the more parsimonious first model. This conclusion is consistent with the AIC and the BIC values for which smaller value is considered as better. That is, the AIC information criterion increased from 9504.7 to 9640.8 , which indicates that model with intercept and slope (both) was a better fitted model (Table 5).

3.1.5.2 Model Selection To select the best model is not possible using only the best mean and variance structure, but also correlation structure. In order to select best variance covariance structure for the final model, first deals with the variance structure by using different variance functions. The combined result was equivalent to dealing with variance covariance structure.

3.1.5.3 Selecting Variance Function for FOTM The variance functions were used to model the variance structure of the within group errors using covariates. The primary tool for investigating within-group Heteroscesdasticity was plots of residuals against the fitted values. Independent variance type of variance function was used by default for this study.

3.1.5.4 Selecting Correlation Structure Function The correlation functions were used to model dependence among observations. Among different correlation structure classes/functions in this study unstructured, compound symmetry, Toeplitz and autoregressive (1) covariance models were used and compared. An information criterion was important to select a responsible covariance structure in order to obtain valid inferences for fixed effects. The small AIC value and the corresponding significant $\mathrm{p}$-value indicated that the model with unstructured covariance function is preferable (Table 6).

After selected the best information criteria based on the smallest AIC with its corresponding P-value, the next step is selection of the best fitted model from homoscedastic and heteroscedastic model. Hence, very small $p$ value corresponding to the likelihood ratio statistics confirmed that the heteroscedastic model explained the data significantly better than the homoscedastic model. The assumption of normality for the within-group errors were assessed with the normal probability plot of the residuals, produced by the 
Table 6 Selection of information criteria to fit responsible structure

\begin{tabular}{lllllrrrr}
\hline Fits & Model & Df & \multicolumn{1}{l}{ AIC } & \multicolumn{1}{l}{ BIC } & \multicolumn{1}{l}{$\log l i k$} & Test & L.Ratio & $p$ value \\
\hline Model. AR(1) & 1 & 19 & 10005.3 & 9997.3 & 9998.3 & & & \\
Model. CS & 2 & 19 & 10106.4 & 10094.4 & 10001.6 & & & \\
Model. Toep & 3 & 19 & 10013.7 & 10013.7 & 9993.6 & & & \\
Model. UN & 4 & 19 & 9504.7 & 9505.1 & 9464.7 & 4 Vs3 & 847.76 & $<0.0001$ \\
\hline
\end{tabular}

Table 7 Selection of best fitted model from homoscedastic and heteroscedastic model

\begin{tabular}{lllllllll}
\hline & Model & Df & AIC & BIC & loglik & Test & L.Ratio & $p$ value \\
\hline Homoscedastic & 1 & 18 & 9684.8 & 9685.4 & 9640.8 & & & \\
Heteroscedastic & 2 & 19 & 9504.7 & 9505.1 & 9464.7 & 1 Vs2 & 84.76 & $<0.0001$ \\
\hline
\end{tabular}

Table 8 Estimates of random effects

\begin{tabular}{lll}
\hline Random effects & SD & Corr \\
\hline Intercept & 7.6189 & ICC \\
time & 5.4086 & 0.635 \\
Residual & 3.1071 & \\
\hline
\end{tabular}

qq normal method. Therefore, for the data set of these types of study heteroscedastic model with unstructured correlation was considered as best final model (Table 7).

Random Effect with positive intercept indicates an increase in BMI of each child provided that time is included in the model. Intra-class correlation coefficient $($ ICC $)=$ $\frac{5.4086}{3.1071+5.4086}=0.635$, indicates that the random effects affects the children BMI data by $63.5 \%$. The residual term indicates that variation with in children in different time of measurements. The term labeled residual is the estimate of $\sigma^{2}$. In addition, random effect with positive intercepts indicates that an increase in BMI each child provided that time was included in the model (Table 8).

$$
\omega=V\left[\begin{array}{l}
b_{i 0} \\
b_{i 1}
\end{array}\right]=\left[\begin{array}{ll}
\omega_{0}^{2} & \omega_{01} \\
\omega_{01} & \omega_{1}^{2}
\end{array}\right]=\left[\begin{array}{ll}
7.6189 & 2.1994 \\
2.1994 & 5.4086
\end{array}\right]
$$

The final model was suggested as:

$$
\begin{aligned}
B M I_{i j}= & 21.501-0.263 T_{i j}-0.101 A_{i}+0 . .550 P_{i}-0.089 M_{i} \\
& +0.658 S F_{i}-0.444 C N O_{i}+0.351 D N O_{i}-0.180 F N O_{i} \\
& -2.523 A R O n_{i}-5.890 A R \operatorname{Pr} e_{i}-0.493 \operatorname{Rrural}_{i}+0.007 P \\
& * T_{i j}-0.122 T_{i j} * S F_{i}+0.0002 T_{i j} * S F_{i} * P+0.042 T_{i j} * M_{i} \\
& +0.008 T_{i j} * A_{i}-1.929 D_{i} * R_{i} * T_{i j}+0.014 B M I_{-1}+\varepsilon_{i j} .
\end{aligned}
$$

The intercept coefficient is $\hat{\beta}_{0}=21.501$ represents an estimate of the average level of children BMI for all covariates. Coefficient of age is $\hat{\beta}_{\mathrm{A}}=-0.101-0.008=-0.109$ indicates that children in the reference group of the mean of BMI decreased by 0.109 
Table 9 First order transition model with main and interaction effect

\begin{tabular}{lclrrll}
\hline Effect & Estimate & SE & DF & t-value & Pr $>|t|$ & $95 \%$ CI \\
\hline Intercept & 21.5009 & 2.0271 & 924 & 10.61 & $*$ & $(20.9162,21.7403)$ \\
Sex Female & 0.6582 & 0.7752 & 924 & 0.85 & 0.3961 & $(-0.0019,0.7093)$ \\
Cough No & -0.4436 & 0.3260 & 924 & -1.36 & 0.1739 & $(-0.5012,0.0019)$ \\
Diarrhea No & 0.3508 & 0.3774 & 924 & 0.93 & $0.0328^{*}$ & $(0.2901,0.3975)$ \\
Fever No & -0.1801 & 0.3116 & 924 & -0.58 & 0.5634 & $(-0.1008,0.0016)$ \\
ART on ART & -2.5234 & 1.1038 & 924 & -2.29 & $0.0225^{*}$ & $(-2.6074,-2.4301)$ \\
ART On pre-ART & -5.8898 & 1.8288 & 924 & -3.22 & $0.0013^{*}$ & $(-5.9910,-5.5001)$ \\
RES Rural & -0.4925 & 0.3237 & 924 & 1.52 & $0.0128^{*}$ & $(-0.5010,-0.4091)$ \\
Age & -0.1009 & 0.0266 & 924 & -3.79 & $0.0002^{*}$ & $(-0.2010,-0.0101)$ \\
MUAC & -0.0885 & 0.1085 & 924 & -0.82 & 0.4145 & $(-0.0991,0.0001)$ \\
PN & 0.0550 & 0.0292 & 924 & 1.88 & $0.0401^{*}$ & $(0.0367,0.0812)$ \\
Time & -0.2628 & 0.5518 & 924 & -0.48 & $0.0234^{*}$ & $(-0.3615,-0.1567)$ \\
PN*Time & 0.0069 & 0.01012 & 924 & -0.69 & $0.0391^{*}$ & $(0.0032,0.0081)$ \\
Time*Sex Female & -0.12174 & 0.4477 & 924 & -0.27 & 0.7858 & $(-0.21674,0.00119)$ \\
MUAC*Time & 0.04240 & 0.03211 & 924 & 1.32 & $0.0471^{*}$ & $(0.02420,0.06147)$ \\
Age*Time & 0.008248 & 0.008716 & 924 & 0.95 & $0.0344^{*}$ & $(0.005919,0.010991)$ \\
PN*Time*Sex Female & 0.00016 & 0.006926 & 924 & -0.02 & $0.0491^{*}$ & $(0.00009,0.00189)$ \\
Diarrhea*Time*RER & -1.9287 & 0.6456 & 924 & -2.71 & $0.0251^{*}$ & $(-2.0001,-1.9987)$ \\
bmi_1 & 0.0143 & 0.0181 & 924 & -0.79 & $0.0427^{*}$ & $(0.0034,0.0267)$ \\
\hline
\end{tabular}

The remaining category of each covariates are reference group

* Statistically significant at $95 \%$ level of confidence level

units per month. Coefficient of plump nut is $\hat{\beta}_{\mathrm{P}}=0.0550+0.0069=0.0619$ indicates that the children in the reference group of the mean BMI increased by 0.0619 units per month. The rate of change of the average children BMI for a unit change of time by the considering the other variables constant is $\hat{\beta}_{\mathrm{T}}=-0.2628$. The average difference of children BMI between having diarrhea disease cases and non-diarrhea is $\hat{\beta}_{\mathrm{D}}=0.3508$. $\hat{\beta}_{\text {on ART }}=-2.5234$ is the average difference of children BMI between on ART and non-ART treatment. The rate of change of average children BMI difference between female who eat plump nut and male who eat plump nut for a unit change of time by considering the other variables constant is $\hat{\beta}_{\mathrm{S} * \mathrm{P} * \mathrm{~T}}=0.00016$. The rate of change of average children BMI difference between no responses who live in rural and positive responses who live in urban through time by considering the other variables constant is $\hat{\beta}_{\mathrm{D} * \mathrm{R}}=-1.9287$. The rate of change of average previous time BMI of children affect, the present time BMI of children by considering the other variables constant is $\hat{\beta}_{\text {bmi-1 }}=0.0143$ (Table 9).

The type-III tests of hypotheses deals about a nice method to decide the final significance covariates in the model. We had retained our significance levels, but it is based on considerably more data. Generally; ART, residence, diarrhea, age, plump nut, time, plump nut over time, MUAC over time, age over time, interaction between plump nut and sex over time, interaction between diarrhea and residence over time and bmi_1 were statistically significance effect on the average BMI of children. The 
Table 10 First order transition model type three tests of hypotheses
* Statistically significant at $95 \%$ level of confidence level

\begin{tabular}{lllll}
\hline Effect & Num.DF & Den.DF & F-value & Pr $>|F|$ \\
\hline Sex & 1 & 924 & 0.72 & 0.3961 \\
Cough & 1 & 924 & 1.85 & 0.1739 \\
Diarrhea & 1 & 924 & 0.86 & $0.0328^{*}$ \\
Fever & 1 & 924 & 0.33 & 0.5634 \\
ART & 2 & 924 & 5.32 & $0.0051^{*}$ \\
RES & 1 & 924 & 2.31 & $0.0128^{*}$ \\
Age & 1 & 924 & 14.33 & $0.0002^{*}$ \\
MUAC & 1 & 924 & 0.67 & 0.4145 \\
PN & 1 & 924 & 3.54 & $0.0401^{*}$ \\
Time & 1 & 924 & 0.44 & $0.0234^{*}$ \\
PN*Time & 1 & 924 & 0.60 & $0.0391^{*}$ \\
Time*Sex & 1 & 924 & 0.07 & 0.7858 \\
MUAC*Time & 1 & 924 & 1.74 & $0.0471^{*}$ \\
Age*Time & 1 & 924 & 0.90 & $0.0344^{*}$ \\
PN*Time*Sex & 1 & 924 & 0.00 & $0.0491^{*}$ \\
Diarrhea*time*RER & 1 & 924 & 0.36 & $0.0251^{*}$ \\
bmi_1 & 1 & 924 & 0.63 & $0.0427^{*}$ \\
\hline
\end{tabular}

remaining covariates were statistically insignificant. But, there is the variation within the subjects. That is. some of the random slops are statistically significance within subjects (Table 10).

\section{Conclusions}

Despite the progress that has been made in the country to improve the problem of children BMI remain high in Bahir Dar Districts. According to this study, there were statistically significant difference among children BMI variation with respect to time, plump nut, age, residence, ART, diarrhea and previous BMI. While; fever, cough, MUAC and sex were statistically insignificant effect on children BMI. According to the main objective of this study results were interpreted as: the mean evolution and variability of BMI were higher on average in females than males. The findings further suggest that if the amount of plump nut were taken by children increase, so the children BMI also increased. Likewise, the interaction between plump nut and female over time had greater BMI than that of the interaction between plump nut and male over time. In addition to that, the average difference of children BMI between children having diarrhea disease cases and non-diarrhea is the coefficient of 0.3508 . Although, children who live in rural area had lower BMI than that of who live in urban area. Even though, children who were on ART had lower BMI than that of who were no ART and children who were pre-ART had similarly lower BMI that of who were no ART children. Finally, the previous children BMI had statistically significant and positive effect on the current children BMI in the first order transitional model. 
Acknowledgements In a special way, I wish to extend my sincere gratitude to Ms. Elesabet G. for the support and guidance she accorded me during this paper. May the God Lord reward her. I would also like to acknowledge the contribution of my colleagues from whom I enjoyed fruitful discussions on challenging topics.

Open Access This article is distributed under the terms of the Creative Commons Attribution 4.0 International License (http://creativecommons.org/licenses/by/4.0/), which permits unrestricted use, distribution, and reproduction in any medium, provided you give appropriate credit to the original author(s) and the source, provide a link to the Creative Commons license, and indicate if changes were made.

\section{Appendix}

\section{See Fig. 4.}

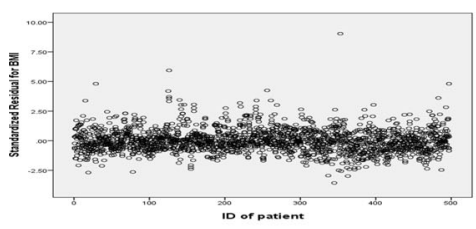

(a)

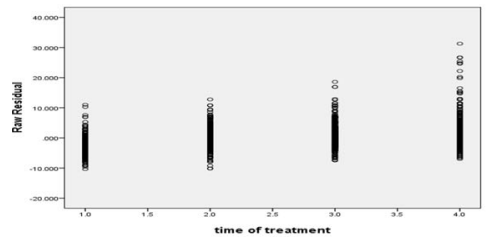

(c)

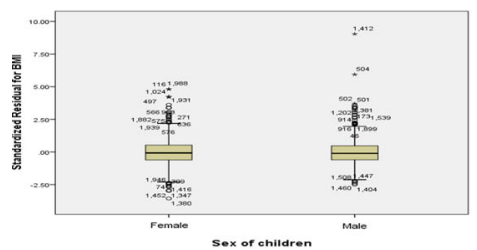

(e)

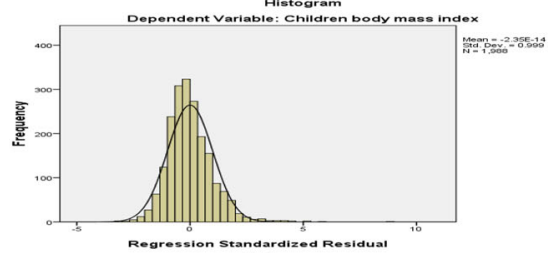

(g)

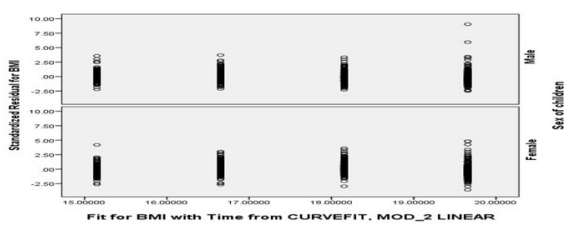

(b)

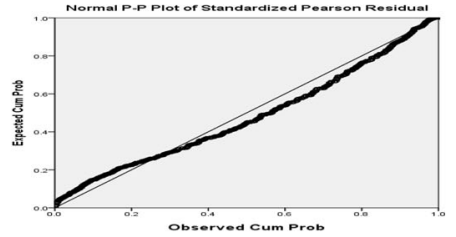

(d)

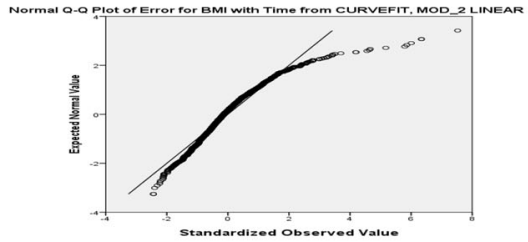

(f)

Fig. 4 a Individual specific residual plots for fitted model, b plots of fitted values versus standardized residuals over sex, $\mathbf{c}$ plot of time versus residuals, $\mathbf{d}$ normal P-P plot observed cum prob versus expected cum prob, e box plot of sex versus standardized residuals, f normal Q-Q plot of standardized observed value vs expected normal value, $\mathbf{g}$ histogram of standardized residual versus frequency of BMI 


\section{References}

1. Adolphe Quetelet (1796-1874) BMI formula was developed. Belgium Statistician. http://www.cdc. gov/healthyweight/assessing/bmi/childrens_bmi/about_childrens_bmi.htm. Accessed 5 July 2012

2. Alemu E, Atnafu A, Yitayal M, Yimam K (2014) Prevalence of overweight and/or obesity and associated factors among high school adolescents in Arada Sub city, Addis Ababa, Ethiopia. J Nutr Food Sci 4:261. https://doi.org/10.4172/2155-9600.1000261

3. Caleyachetty R, Rudnicka AR, Echouffo-Tcheugui JB, Siegel KR, Richards N, Whincup PH (2012) Prevalence of overweight, obesity and thinness in 9-10 year old children in Mauritius. Glob Health $8: 28$

4. Dietz W (1995) Does hunger cause obesity? Pediatrics 95(5):766-767

5. Diggle PJ (2002) Analysis of longitudinal data, 2nd edn. Oxford University Press, New York

6. Ferro-Luzzi A, Sette S, Franklin M, James WP (1992) A simplified approach of assessing adult chronic deficiency. Eur J Clin Nutr 46:173-186

7. Gebregergs GB, Yesuf ME, Beyen TK (2013) Overweight and obesity, and associated factors among high school students in Gonder Town, North West Ethiopia. J Obes Weight Loss 3:165. https://doi.org/ $10.4172 / 2165-7904.1000165$

8. Gurrin LC et al (2005) Solving mixed model equations, vol 120. Wiley, New York, pp 120-142

9. Henderson CR (1984) Applications of linear models in animal breeding. University of Guelph Press, Guelph

10. Kassahun T (2013) Semi-parametric analysis of children nutrition status in Ethiopia. Int J Stat Appl 3(5):141-154. https://doi.org/10.5923/j.statistics.20130305.01

11. Laird NM, Ware JH (1982) Random-effects models for longitudinal data. Biometrika 38:963-974

12. Poirier P, Giles TD, Bray GA, Hong Y, Stern JS, Pi-Sunyer FX, Eckel RH (2006) Obesity and cardiovascular disease: pathophysiology, evaluation, and effect of weight loss. Circulation 113:898-918

13. Salman Z, Kirk GD, Deboer MD (2011) High rate of obesity-associated hypertension among primary schoolchildren in Sudan. Int J Hypertens 5:169-173

14. Singer JD (1998) Using SAS Proc Mixed to fit multilevel models, hierarchical models, and individual growth models. J Educ Behav Stat 23:323

15. Tango $\mathrm{T}$ (2016) On the repeated measures designs and sample sizes for randomized controlled trials. Biostatistics 17(2):334-349

16. Verbeke G, Molenberghs G (2009) Linear mixed models for longitudinal data. Springer, New York, NY

17. WHO (2012) Info base data on overweight and obesity, mean BMI, healthy diets and physical inactivity. Geneva

18. WHO Media Center fact sheet (2013) Obesity and overweight

Publisher's Note Springer Nature remains neutral with regard to jurisdictional claims in published maps and institutional affiliations. 